\title{
DASSARETIA ET PENESTIANAE TERRAE À LA LUMIÈRE DES DONNÉES ONOMASTIQUES
}

\begin{abstract}
Ancтракт. - Ономастиката беше една од дисциплините што во текот на својата плодна кариера ги обработуваше проф. Фанула Папазоглу на која ѝ е посветен овој труд, а по повод стогодишнината од нејзиното раѓање. Со методолошкиот пристап на распоредување имиња во помали, јасно ограничени и затворени подрачја ги постави темелите за изучување на сложената етничка и социјална структура на Балканот во античко време, област во која неприкосновено владееше, а се истакна и на меѓународен план. Признание за овој нејзин придонес ѝ беше оддадено во далечната 1977 год. со поканата за воведен реферат на седмиот меѓународен епиграфски конгрес чија главна тема беше ономастиката. Од нејзиното перо е и првиот труд за ономастиката на дасаретските племиња во римско време, при што и самата објавуваше натписи од Охрид. Стогодишнината од нејзиното раѓање за мене беше повод повторно да се навратам на дасаретската ономастика, дотолку повеќе што во меѓувреме бројот на натписи во Дасаретија е зголемен.
\end{abstract}

L'onomastique était un des domaines de recherche du prof. F. Papazoglou qu'elle dominait avec maîtrise. L'application de la méthode de classification des noms par microrégions onomastique s'est avérée acceptable et très utile pour l'étude de la structure ethnique et sociale des peuplades dans les Balkans, et c'est avec les résultats de ses recherches que le VIIe congrès épigraphique a été inauguré. ${ }^{1}$ De sa plume est également issu le premier travail sur l'onomastique des Dasarètes à l'époque romaine. ${ }^{2}$ Le $100^{\mathrm{e}}$ anniversaire de sa naissance est une occasion de revenir sur ce sujet et de repenser la structure ethnique que j'avais moi-même abordée. ${ }^{3}$ La reprise du sujet se justifie d'autant plus que depuis, le nombre des inscriptions découvertes dans la partie occidentale de

${ }^{1}$ Structure ethnique et sociale dans les Balkans, à la lumière des études onomastiques, Actes du VII, congrès internationale d'épigraphie grecque et latine, 1977, Bucarest-Paris, 1979, pp. 151-169.

2 Охридско во праисторијата и во античкиот период, in Охрид, vol. I, Скопје, 1985, pp. 63-124, surtout pp. 101-113.

${ }^{3}$ N. Proeva, Enchéléens, Dassarètes, Illyriens, les sources littéraires, épigraphiques et archéologiques, in L'Illyrie méridionale et l'Epire dans l'Antiquité, II Clermont-Ferrand 1990, Paris 1993, p. 197 sqq.; Ead., Студии за античките Македонци, Скопје 1997 (résumé 267-278), pp. 137-147. 
la République de Macédoine s'est accrue et que les inscriptions connues ont été révisée et republiée avec des corrections. ${ }^{4}$ De même, la publication du corpus des inscriptions des deux colonies grecques Apollonia et Dyrrhachion et de la ville de Bouthrotos sur le littoral albanais ${ }^{5}$ qui manquait cruellement, nous permet de comparer l'onomastique de ces régions voisines et de mieux étudier les noms considérés comme illyriens sur lesquels les opinions sont loin d'être unanimes. Il existe toujours un certain nombre de noms rares ou uniques difficiles à classer et la notion «illyrienne» reste toujours floue. De plus, il faut tenir compte des relations existantes entre l'Illyrie méridionale et la Macédoine, qui ont eu un tel impact sur l'onomastique qu'il n'est pas facile de discerner les noms véritablement illyriens dans le sens des Illyrii proprie dicti. Les noms macédoniens ne sont pas facilement identifiables non plus, en raison de leur classement dans le répertoire grec pris dans le sens le plus large, ce qui fausse les proportions entre ces trois groupes de noms, d'autant plus que la plupart des inscriptions datent de l'époque romaine. Seul un classement géographique rigoureux par régions bien délimitées (méthode de Namengebiet) ${ }^{6}$ peut aboutir à des résultats plus exactes. Bien qu'aujourd'hui on puisse plus nettement discerner la structure de l'onomastique illyrienne, la survivance de l'ancienne théorie pan-illyrienne pèse encore sur les recherches onomastiques.

Géographiquement, la partie occidentale de la République de Macédoine, surnommée par chercheurs «la partie illyrienne de la Ma-

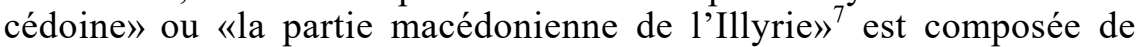
trois régions qui sont, du sud au nord: le bassin du lac d'Ohrid (Lychnites limne) avec les villes Ohrid et Struga (R. de Macédoine), et les villes Podgradec (Pogradeci) et Gorica (Koritsa/Korçë), aujourd'hui en Albanie; la région au cours supérieur de la rivière Treska avec ville de Kičevo) et la plaine de Polog au nord, qui englobe les villes actuelles de Gostivar et de Tetovo. La partie sud-ouest (bassin du lac d'Ohrid) était considérée par la plupart des chercheurs comme illyrienne ${ }^{8}$ et

\footnotetext{
${ }^{4}$ Seules les inscriptions du bassin d'Ohrid y sont incorporées, IG X, II, 2. $n^{\circ} 352-$ 411 , celles des deux régions au nord, quoique peu nombreuses n'y sont pas insérées, ce qui est regrettable. A défaut de corpus des inscriptions de la partie albanaise de la Dassarétie (la ville de Podgradec et Korçë), il est possible que certaines inscriptions m'aient échappées, mais leur nombre ne devrait pas être significatif au point d'altérer mes conclusions.

${ }^{5}$ CIGIME, vol. 1,1, 1995 Epidamne- Dyrrhachion; vol 1.2, 1997 Apollonia; vol. 2.22007 , Boutrothos.

${ }^{6}$ Appliqué par R. Katičić pour la région illyrienne pris dans le sens le plus large de province primordiale.

${ }^{7}$ Il est notoire que les Romains avaient rattaché l'Illyrie méridionale à la province de Macédoine. Les raisons en étaient: la nécessité d'éviter les barrières sur la route Via Egnatia, de même que l'appartenance de l'Illyrie du sud à la zone culturelle orientale, dont la langue de communication était le grecque.

${ }^{8}$ A. Philipson, Dasaretis, in RE IV, col 2221; N. G. L. Hammond, Epirus, Oxford, 1967, P. Cabanes, L'Epire, Paris, 1967; F. Papazoglou, L'organisation politique de l'Il-
} 
la partie nord-ouest (région de Kičevo et de Polog) comme dardanienne, appartenant à la Mésie Supérieure, mais les autres opinions ne manquent pas. ${ }^{9}$

Les maigres données chez les auteurs anciens sur l'appartenance ethnique des tribus habitant cette partie frontalière ne sont pas claires, car les mentions des ces auteurs concernent plutôt les relations politiques des ethnies et leur organisation administrative - notamment ils employaient les dénominations ethniques souvent selon l'appartenance à une unité politique. Cependant, grâce aux données onomastiques dont on dispose maintenant, les perspectives se sont améliorées et on peut étudier ces questions avec beaucoup plus de facilité et avec moins de conjectures.

De l'époque romaine à la fin de l'antiquité, le nom de Dassarétie s'est imposé pour les dîtes trois régions. ${ }^{10}$ Pline le Jeune nous rapporte que les Dassarètes s'étendaient au dessus de (a tergo suo... supra) de l'Epire jusqu'aux farouches (mox feram) Dardaniens, ${ }^{11}$ ce qui est en accord avec le donnée de Strabon qui les place à côté de Ardiéens et Dardaniens,${ }^{12}$ de même quand il écrit au sujet de la frontière entre Macédoniens et les Illyriens. ${ }^{13}$ Le pays d'origine des Ardiéens, avant leur extension à l'ouest jusqu'au Rhizonicus Sinus (aujourd'hui golfe de Boka Kotorska en Monténégro) vers la fin du IV s., était dans la région montagneuse de Monténégro. ${ }^{14}$ Après les deux guerres illyro-romains, les Illyriens au sud de Lissos en étaient sous la domination romaine et seuls les Ardiéens avaient sauvegardé l'indépendance. ${ }^{15}$ Parmi les éthnies puissantes qui ont disparues ou qui étaient alors afaiblies Strabo mentione les Ardiéens qui étaient en lute avec les Autariates pour des

lyrie méridionale, à propos du livre de $\mathrm{P}$. Cabanes, Les Illyriens de Bardylis à Genthios, ŽAnt, XXXIX, Skopje, 1989, p. 31-53, avec renvoi à ses travaux précédents sur le sujet.

${ }^{9}$ La revu de différentes opinions chez N. Proeva-O. Brankoviḱ, Le problème de localisation de la ville d'Uscana en Haute Dassarétie, in L'Illyrie méridionale et l'Epire dans l'Antiquité, IV Grenoble, 2002 [2004], p. 197; Н. Проева, За дасаретските племиња (Penestae, Dasaretae, Engeleanes) и местоположбата на градот Uscana во Горна Дасаретиda, ŽAnt, LXIV, 2014, p. 166/7.

${ }^{10}$ N. Proeva, Enchéléens, Dassarètes, Illyriens, p. 197 sqq.

${ }^{11}$ Plinius, HN, IV, 3. A cette époque les Dardaniens occupaient les plaines de Kosovo, Metohija et de Skopje.

${ }^{12}$ Strabo, VII, 7, 5, 12 (C 318) Selon F. Papazoglu, Srednjobalkanska pelemena u predrimsko doba, Sarajevo, 1969, p. 221, n. 47 cette mention est surprenante.

${ }^{13}$ Strabo, VII, 7, 5, 2 (C 316). Cette phrase est deformée (F. Papazoglou, Plemena, p., 77, 274, n. 263) et si on la compare avec l'autre mention de Strabo, ainsi qu'avec celle de Pline il est évident qu'il faut corriger le nom d'Autariates en Ardiéens, ce qui rend la phrase compréhensible.

${ }^{14}$ Ф. Папазоглу, О територији илирског племена Ардиеја, Зборник Филозофског факултета, Београд, 1963, VII/1, p. 71-86, (résumé 84-86).

15 М. Гарашанин, Историјска и археолошка разматрања о илирској држави, Глас САНУ CCLX, Београд, 1974, стр. 6 -28. (résumé 29-32). 
sources salées ${ }^{16}$ ensuite ont été pressés par les Macédoniens et les Romains. ${ }^{17}$ Cela démontre bien que les Ardiéens étaient les voisins des Macédoniens, c'est à dire des Dassarètes. Ces données qui étaient négligés dans la discussion sur le territoire de Dassarètes nous autorisent à utiliser les dénominations Dassarétie méridionale et septentrionale pour cette vaste région. Dans la partie nord-ouest de Macédoine, la Dassarétie du nord, d'après Pline le Jeune, la seule tribu mentionnée par T. Live est celle des Pénestes (Penestai), ${ }^{18}$ à propos de l'opération militaire entreprise par le roi Persée en 170 av. n. ère, afin de protéger la frontière nord du royaume macédonien. ${ }^{19}$

Dans la région méridionale de Dassarétie, le bassin du lac d'Ohrid, les sources mentionnent d'abord les Enchéléens, les Bryges, les Sarnoates, les Sessarethoi (Dessaretoi ?), les Dassarètes, les Pisantinoi, Kaloikinoi, ainsi que le nom du lac Lychnites limne (et de la région Lychnis), qui apparaît au milieu du IV s. av. n. ère et celui des Dassarètes vers la fin du III s. La ville de Lychnidos (actuelle ville d'Ohrid) ${ }^{20}$ était le siège du koinon des Dassarètes ${ }^{21}$ et fut florissante jusqu'à la fin de l'Antiquité.

Sur l'appartenance ethnique des Dassarètes, comme sur celle des Engelanes, la tribu premièrement attestée dans la Dassarétie du sud, ${ }^{22} \mathrm{ni}$ les savants modernes, ni les auteurs anciens, ne sont unanimes. La dénomination de ces derniers variait selon la situation politique. Les auteurs anciens les mentionnent tout à la fois comme étant des Illyriens mais également différents de ceux-ci. Ils sont distingués par Tite

${ }^{16}$ Ps. Aristoteles, de ausc. mir., 138.

${ }^{17}$ Strabo, VII, 5, 6 (c 315): «...hysteron d'ypo Makedonon kai Romaion ekpolemoumenoi. » Cela est confirmé par Polybios, VIII, 14 : Philippe V, après s'être emparé de Lissos en 214 avait occupé les environs et la plupart des Illyriens lui avaient cedé leurs villes sans garder une seule forteresse, ce qui montre que les Ardienes etaient sous la domination macédonienne, en 209 il lui a été réclamé leur restitution (T. Liv., XXVII, 30,13).

${ }^{18}$ T. Livius, XLIII, 19, 5.

${ }^{19}$ T. Livius, XLIII, 10, 1.

${ }^{20}$ N. Proeva, Lychnidos-Loukridon-Lokrida-Ohrid, in L'lllyrie méridionale et l'Epire dans l'Antiquité, III Paris 1996, p. 73-78; Ead., Sur la localisation de la ville de Lychnidos et sur l'origine du nom de la ville d'Ohrid, Antidoron M. Suić, Zagreb/ Zadar 2015, sous presse.

${ }^{21} I G$ X, II, 2 Lychnidos, $n^{\circ} 373,378,379$; Dassaretioi ${ }^{\circ} 360,362,368,369,370$, 371, 372. Le koinon frappait des monnaies de deux type différents. L'un avec tête de Zeus/ Amon au recto, au revers foudre entre la légende $\triangle \mathrm{A} \Sigma \Sigma \mathrm{A} / \mathrm{PHTI} \Omega \mathrm{N}$, le tout dans la couronne de chêne. L'autre type diffère au recto - la tête d'Héraclès avec peau de lion est figuré. A nos jours on a trouvé 4 exemplaires, datés du II s. av. n. ère, l'un dans la nécropole de Deboj près d'Ohrid et trois à Ohrid (loc. Plaošnik), cf. Размовска - Бачевска, Монетоквањето во Охрид (Lychnidos) II век пр. н.е. - XIII-XVII век (summary), Ohrid, 2016, type I-II, 6-7, 26.

${ }^{22}$ Voir la discussion chez N. Proeva, The Engelanes/Encheleis and the golden masks from Trebenishte culture, in Homage to Milutin Garašanin, Belgrade, 2006, pp. 561-570, Ead. N. Proeva, La coutume funéraire des masques d'or en Macédoine archaïque, in Maschere, teatro e rituali funerari nel mondo antico, Roma, 2017, à paraître. 
Live $^{23}$ et Pomponius Mella. Ce dernier les mentionne à coté de ceux qui «proprie Illyrios vocant», ${ }^{24}$ ce qui montre que les Dassarètes, ainsi que les Engelanes, étaient les voisins d'Illyrioi proprie dicti, et que le noyau de l'état illyrien n'était pas dans la région d'Ohrid comme certains le pensent. ${ }^{25} \mathrm{Ce}$ voisinage a contribué à les inclure dans le groupe des tribus illyriennes, malgré le fait qu'ils ne fussent pas des Illyriens à proprement parler. L'onomastique, comme on va démontrer, témoigne que ces tribus n'étaient pas des Illyriens. Il faut noter que la densité des inscriptions dans ces trois régions est inégale, ce qui est dû aux degrés différents des recherches effectuées.

L'analyse onomastique pour la Dassarétie méridionale est basée sur les 50 noms "grecs» des indigènes figurants sur les inscriptions, et deux gravés sur des objets (un casque et une bague), attestés en majeure partie dans la région du lac d'Ohrid. Y sont attestés encore les 16 prénoms et cognomens romain ainsi que le 8 gentilices.

Le nombre important de noms macédoniens (Alexandros, Amyntas, Kassandra, Kleopatros, Krates, Lennatos, Lysimachos, Machatas, Ptolemaios, Philippos) serait surprenant si l'on présume une origine illyrienne de ces tribus. Les autres noms sont typiques de la zone culturelle orientale, surtout d'Asie Mineur. Parmi tous ces noms seuls six noms: Annia, Genthios, Dazos, Epikados, Plator et Preurados et deux (Monunios et Tata) gravés sur des objets, sont d'habitude considérés comme illyriens.

Annia apparaît comme nom et comme gentilice. Le nom appartient à la famille onomastique d'Anna, avec ou sans gémination, y compris les variantes avec différents suffixes (-aios, -aia, -oula, -ula respectivement -aius, -aea, -ula, et -aeus, -eus). Ils sont d'une parfaite banalité, on les trouve dans l'onomastique de tous les peuples, le plus souvent en Asie Mineure. ${ }^{26}$ Ces noms sont répandus aussi chez les Sémites bien qu'il soit difficile de savoir si le nom est sémitique d'origine ou tout simplement adopté par des Sémites hellénisés. Ce qui est important pour notre propos ici, c'est le fait que la forme Annia n'est pas attestée en Illyrie du sud. Par contre, dans la région d'Ohrid, Annia l'est deux fois comme nom propre. Une fois sur l'inscription

${ }^{23}$ T. Livius, XLII, 36, 8-9: inde tribunos cum duobus millibus militum ad occupenda Dassaretiorum et Illyriorum castella, ipsis arcessentibus praesida, ut tutiores a finitimorum impetu Macedonum essent, misit.

${ }^{24}$ P. Mella, II, 54-56. Strabo, VII, 5.1 utilise la dénomination Ilyrica Prota.

${ }^{25}$ Le plus probable est que les vrais Illyriens, Illyrioi proprie dicti, habitaient sur la côte entre l'Epidamnos et Lissos comme il est soutenu par R. Katičić, Illyrii proprie dicti, ŽAnt, XIII/XIV, 1964, p. 87-97; rejeté par F. Papazoglou, Les origines et la destinée de l'Etat illyrien: Illyrii proprie dicti, Historia, XIV/2, 1965, p. 177-179, qui pense qu'ils devraient être plus près de la Macédoine; M. Suić, Illyri proprie dicti, GodCBI, XII, 1975, p. 179-196, les localise dans la région de Lissos, en Albanie septentrionale.

${ }^{26}$ L. Zgusta, Kleinasiatishe Personennamen, Prag, 1964, no. 62-12. 
métrique d'une certaine Annia pour son fils Demokritos, ${ }^{27}$ une autre fois Annia est fille de Plator, dont les enfants et le mari portent des nomes typiques pour la Macédoine (Alexandros, mari et un des fils, Ariston et Neikopolis). ${ }^{28}$ Et c'est le nom Plator, le patronyme d'Annia qui appelle un commentaire. Dans la partie albanaise de Dassarétie, on trouve ce nom encore deux fois: une fois à Podgradec ${ }^{29}$ et une fois à Korçë. ${ }^{30}$ Un Plator d'Orestide est mentionné par Cicero. ${ }^{31}$ Par contre, en Illyrie méridionale, en tant que nom ou comme patronyme, n'est attesté que dans les colonies grecques d'Epidamnos et d'Apollonia ${ }^{32}$ et très rarement dans l'arrière pays. ${ }^{33}$ A Delphes un Plator, est désigné genos italos,${ }^{34}$ les formes messapiennes Plator, Platura sont connues, ${ }^{35}$ et le gentilice Platorius ${ }^{36}{ }^{36}$ est attesté partout dans les monde romain. Le nom est relativement répandu dans le monde $\operatorname{grec}^{37}$ (Epire, Thessalie, Béotie, Locride) et dans la plupart des cas dans un contexte servile. Il faut évoquer encore la glose d'Hesichios «platur: doulos e demos» pour montrer que ce nom et ses variantes ne sont pas illyriens d'origine mais simplement à la mode chez eux. $\mathrm{C}$. De Simone classe ces noms dans le groupe non sud-illyrien. ${ }^{38}$

Le nom masculin Genthios au génitif, en tant que patronyme d'un Machatas, est attesté sur l'inscription du village de Kalište, au bord du lac d'Ohrid, ${ }^{39}$ dans un contexte de mixité qui présente un mélange de noms macédoniens, (Philippos, Nikias), romains (Mountanos,

${ }^{27}$ Dans la dernière édition de l'inscription IG X. II, 2, no 386, la lecture est corrigée de telle sorte que le nom Olbiste est supprimé.

${ }^{28} I G, \mathrm{X}, \mathrm{II}, 2$ no 383.

${ }^{29} \mathrm{~V}$. Toçi, Données sur l'élément illyrien à Dyrhachium à la lumière des nouveaux témoignages archéologiques, Studia Albanica, 9/1, 1972, p. 84.

${ }^{30}$ P. Lera, Phithoi antiques munis d'estampilles découvertes dans le district de Korçë (titre du résumé), Iliria, 2, 1983, p. 218, no 3.

${ }^{31}$ Cicero, De har. Resp., 35.

${ }^{32}$ CIGIME, I, 1, nos 229, 256, 276, 352-356, 375, 429; I, 2 nos 65 et 159, avec des noms pas typiquement illyrien.

${ }^{33}$ Un fois à l'extrémité de Dassarétie, à Elbasan (V. Toçi, Données sur l'onomastique illyrienne à Dyrrhachium et dans d'autres centres de l'Albanie, Studia Albanica, 1969, p. 175), et un Plator de Dimale était proxène à Oropos (IG VII, 282 ).

${ }^{34}$ H. Collitz-F. Bechtel, Sammlung der griechischen Dialekteninschriften, Göttingen 1884 , no 1800 .

${ }^{35}$ C. De Simone, Iscrizioni messapiche della Grotta della Poesia, Annali dela Scuola Normale Superiore di Pisa, XVIII/2, Pisa, 1988, p. 328/9 et 377.

${ }^{36}$ Il semble être d'origine étrusque: Splatur, cf. W. Schultze, Zur Geschichte lateinische Eigenamen, $1966^{2}$, p. 262, 335, n. 4.

${ }^{37}$ Les exemples de Plator dans le monde grecque, réunis par L. Robert, Noms indigènes dans l'Asie gréco-romaine, Paris, 1963, p. 414, et par O. Masson, Variétés thessaliennes, $R P h$, LIV/2, 1980, p. 229-230.

38 cf. L'elemento non greco nelle iscrizioni di Durazzo ed Appolonie, in Grecs et Illyriens p. 45 app. C (nomi non sud orientali) et p. 72/3.

${ }^{39} I G, \mathrm{X}, \mathrm{II}, 2$ no 367. 
Leukios, probablement transcription grecque de Lucius) et un nom supposé illyrien (Epikados). Genthios est considéré comme illyrien, mais le nom et ses formes apparentes manifestement grécisées (Gentheas, Gentheis, Genthion, Genthon) sont fréquents dans bien des régions du monde grec, y compris les deux cités grecques sur la côte illyrienne, et très rares dans la zone illyrienne du nord. Par contre, il est attesté en Italie $^{40}$ et dans les provinces romaines et comme gentilice (Genthius, Gentilius) et comme cognomen (Gentilis, Gentillus, Gentinus, Gentius, Gentus). ${ }^{41}$ Genthios, ${ }^{42}$ ainsi que Gentianus ${ }^{43}$ en tant que cognomen chacun, une fois ont été trouvés à Apollonia. Il faut souligner que ces noms ne sont pas attestés dans l'arrière pays des deux cités grecques. En Macédoine, un castel au nom Gentianon est mentionné par Proco$\mathrm{pe}^{44}$ sans plus de précision. Il est probable qu'il se trouvait en Dassarétie, car il est mentionné entre les toponymes Parthion, évidemment parthinienne et Priniana, toujours non identifié. Cela d'autant plus que Pline le Jeune localise les Dassarètes dans l'arrière pays de Parthines et des monts Candaviens. ${ }^{45}$ Pour sa part, Pline le Jeune ${ }^{46}$ écrit que «Gentius (sic), roi des Illyriens, a découvert la gentiane; elle crô̂t partout, mais la plus estimée est celle de l'Illyrie». Cela explique l'apparition tardive du nom (ce roi régnait au II s. av. n. ère), ainsi que la popularité de ce nom et de ses variantes chez les Illyriens hellénisés Genthios est attesté comme le magistrat monétaire à Dyrrhachion. ${ }^{47} \mathrm{Il}$ faut aussi souligner que les autres noms dynastiques chez les Illyriens, comme Agrôn, Ballaios, Bardylis, Grabos, ${ }^{48}$ Jonios etc, à la différence de noms dynastiques macédoniens, ne sont pas attestés épigraphiquement, car ils ne sont pas illyriens d'origine mais acceptés par la couche dirigeante et hellénisée. Cela est encore un autre argument en faveur de l'origine non illyrienne de ces noms, en usage dans de nombreuses régions du monde grec.

Dazos est un nom qu'on trouve partout dans la zone de langue grecque (de Delphes au Rhénée) très souvent avec les ethniques d'Ita-

${ }^{40}$ Les exemples rassemblés par R. Katičić, Illyrische Personennamen im ihrem südöstlischen Verbreintungsgebiet, ŽAnt, XII, 1962, p. 105-106.

${ }^{41}$ A. Mocsy, Nomenclator provinciarum Europae latinarum et Galliae Cisalpinae, Budapest, 1983, Diss. Pann. III/1, s.v.

${ }^{42}$ CIGIME, I,2, no 212.

${ }^{43}$ CIGIME, I,2, no 189.

${ }^{44}$ Procopius, De aedificiis, IV, 4.

${ }^{45}$ Plinius, HN, III.145, 5: «Gentes Partheni et a tergo eorum Dassaretae, montes Candaviae a Dyrrachio ».

${ }^{46}$ Plinius, NH, XXV, 71. Dioscurides Pedianus, de Cilicie qui a travaillé comme médecin militaire à Rome va plus loin en disant que la plante était nommée selon le nom du roi, évidement c'est l'envers qui est vrais.

${ }^{47}$ CIGIME I, 1 et I, 2. s.v.

${ }^{48}$ A Appolonia Grabos est attesté en tant que monétaire, CIGIME, no 170, et une fois Agron (père de Neikaia), no 126. Selon L. Robert L. ces deux noms sont ioniens, Monnaies grecques, Paris, 1969, pp. 26-28. 
lie du sud, car il s'agit d'un nom messapien grécisé ${ }^{49}$ et répandu dans les régions occidentales (Epire, Acarnanie, Thessalie), ainsi qu'en Asie Mineure, ou il est considère comme épichorique. ${ }^{50}$ Il n'y en a pas trace en Illyrie du sud. Dans les deux colonies grecques sur le littoral, on trouve souvent ses formes dérivées, telles Dazios, Dazaios, Dazeis, Dazeion etc, si bien qu'on les considère comme illyrien. ${ }^{51}$ Selon C. de Simone le nom a pu être répandu par les marchands ou les esclaves ${ }^{52}$ mais il se peut que cela révèle plutôt la parenté avec les tribus messapiennes. En Dassarétie, un Dazos, dont le père porte le beau nom macédonien Lennatos, apparaît une fois à Ohrid, ${ }^{53}$ et la forme Dazeion, identique au prénom messapien à Podgradec. ${ }^{54}$

Epikados. C'est un des noms les plus fréquents dans les colonies grecques d'Apollonia et de Dyrrhachion, à Bylis un gladiateur le porte ${ }^{55}$ et il est également répandu en Epire et en Dalmatie. Le nom est considéré et comme grec (prefix $-e p i)^{56}$ et comme illyrien. Selon Katičić, le nom provient du messapien, ${ }^{57}$ selon d'autres ${ }^{58}$ le nom se serait répandu de l'Illyrie méridionale vers l'Italie. Ceci est tout de même moins vraisemblable, vu la présence d'autres noms d'origine messapienne chez les Illyriens du sud, et montre plutôt la corrélation entre le messapien et l'illyrien. Epicados, est attesté une fois à Orestide ${ }^{59}$ et cinq fois sur les stèles éphébiques de Styberra en Derriopos, datant du I siècle de $\mathrm{n}$. ère et les deux premières décennies du II. ${ }^{60}$ Le nombre de 284 personnes est impressionnant, et la prépondérance des noms macédoniens remarquable. Or, il est invraisemblable que des personnages portant ce nom aient été des immigrés (d'ailleurs les trois éphèbes étrangers domiciliés à Styberra sont désignés par leurs ethnies). Cela démontre que tous ses Epicados ne sont pas illyriens. ${ }^{61}$ En Dassarétie le

${ }^{49}$ C. De Simone, Grecs et Illyriens, p. 38-39.

${ }^{50}$ L. Zgusta, op. cit., p. 245.

${ }^{51} \mathrm{On}$ a des exemples de ces noms avec la dénomination illyrienne: Dazis Illyria à Démétrias cf. Bul.ép., 1958, no 301, p. 266.

${ }^{52}$ C. De Simone, Grecs et Illyriens, p. 39.

${ }^{53} I G, \mathrm{X}, \mathrm{II}, 2$ no 396.

${ }^{54}$ V. Toçi, Studia Albanica, 9/1, 1972, p. 84.

${ }^{55}$ P. Sestieri, Iscrizioni latine d'Albania, Tirana, 1942, no 20.

${ }^{56}$ F. Bechtel, Die historischen Personennamen des griechischen bis zur Kaiserzeit, Hale, 1917, p. 157.

${ }^{57}$ R. Katičić, ŽAnt, XII, 1962, p. 101-103.

${ }^{58} \mathrm{M}$. Beauregard, L'apport des monnaies à l'étude de l'onomastiques d'Apollonia d'Illyrie et d'Epidamne- Dyrrrhachion, in P. Cabanes (Dir.). Grecs et Illyriens dans les inscriptions en langue grecque d'Epidamne-Dyrrhachion et d'Apollonia d'Illyrie, Acte de la Table ronde internationale, Clermont-Ferrand 1989 (Paris 1993), p. 102, n. 99.

${ }^{59}$ EAM, no 186.

${ }^{60} I G$ X, II, 2 nos 323, 324, 325, 326.

${ }^{61}$ Il est possible que ce nom recouvre des noms indigènes semblables. Il existerait une graphie d'Ephikados avec « fi », si la lecture du timbre d'un pythos trouvé à Podgradec est bonne cf. V. Toçi, Données sur l'onomastique illyrienne à Dyrrhachium et dans d'autres centres de l'Albanie, Studia Albanica, 6/1, 1969, p., 171, n. 21, et p. 177 (l'auteur n'as pas donné de photo). 
nom n'est repéré qu'une seule fois, ${ }^{62}$ ensemble avec les noms Preurados et Amnia, ce dernier étant non attesté en Macédoine.

Quant à Preurados ce nom est absent chez les Illyriens ordinaires, à l'exception de trois exemples à Dyrrhachion. ${ }^{63}$ Le nom est connu sous la forme grécisée Pleuratos, et sa transcription latine Pleuratus, comme le nom d'un roi des Ardiéens. ${ }^{64}$ Hormis une unique attestation en Dassarétie ${ }^{65}$ la forme Preurados est fréquente en Macédoine. La graphie Preuratos apparaît sur trois tuiles trouvées à Lyncestide. ${ }^{66}$ La même apparaît à Orestide: à Battyna, ${ }^{67}$ à Lyke dans un contexte purement macédonien, ${ }^{68}$ et sur une tuile, datée du II s. av. n. ère, de l'île Golem Grad, lac de Prespa. ${ }^{69}$ La graphie Pleuratos ${ }^{70}$ est attestée deux fois à Berroia - l'une étant la dédicace d'Héraclès Kynagidos, ${ }^{71}$ et l'autre une inscription incomplète. Le nom est formé avec le sufixe -atos (comme le macédonien Leonnatos/Lennatos) qui existe aussi dans l'onomastique phrygienne et qui nous montre l'origine brygienne de ce nom. Cela veut dire qu'on ne peut pas accepter l'opinion selon laquelle il s'agit d'une influence de l'onomastique illyrienne en domaine macédonien, ${ }^{72}$ d'autant plus que la fréquence de ce nom en Macédoine dépasse de loin les trois exemples attestés chez les Illyriens, plus exactement dans la colonie grecque de Dyrrhachion.

De deux noms gravés sur des objets celui de Monunios gravé sur un casque du type bonnet phrygien découvert sur les bords du lac d'Ohrid, est mentionné comme le nom du roi illyrien en 280 av. n. ère, ${ }^{73}$ ce qui en constitue le seul témoignage littéraire. Dans l'onomastique illyrienne il est très rare: hormis sur les monnaies du roi, le nom est porté par un monétaire de Dyrrhachion. ${ }^{74}$ La seule attestation épigraphique est une stèle d' Apollonia, perdue entre temps. ${ }^{75}$ Mais le plus ancien témoignage littéraire du nom date du IV $s$. avant $n$. ère: c'est le nom du frère du Ketriporis, roi d'Odrisiens. Le nom figure sur une stèle funéraire à Amphipolis, avec le beau nom macédonien - Amyntas. Comme on le croit illyrien, la présence de ce nom chez les Odrisiens était expliquée comme conséquence d'un mariage dynastique (mais il

${ }^{62} I G \mathrm{X}$, II, 2, no 407.

${ }^{63}$ CIGIME, I, 1 nos 48, 106, 364.

${ }^{64}$ Polybios, II, 2; T. Livius, XXVII, 30.

${ }^{65}$ IG X, II, 2, no 407.

${ }^{66} E A M$, no 179 .

${ }^{67} E A M$, no 195, stèle funéraire; sur le décret de Battyna, no 176 il s'agit d'émendation du nom.

${ }^{68}$ J. et L. Robert, Bul Ep. 1964, no 242.

${ }^{69}$ V. Bitrakova -Grozdanova, Golem grad, Prespa. Vol. I, Skopje, 2011, p. 201.

${ }^{70}$ Bul.ép., 2002, no 47.

${ }^{71}$ EKM, nos 30 et 141.

${ }^{72} \mathrm{O}$. Masson, A propos de la réimpression des Beamtennamen auf des griechishen Munzen de Rudolf Munsterberg, RPh, 103, 1977, p. 87.

${ }^{73}$ Trogue-Pompée, XXIV, prologue.

${ }^{74}$ M. Beauregard, op. cit., p. 103.

${ }^{75}$ CIGIME, no 124. 
n'y a aucun indice à ce sujet), et celui d'Amphipolis comme étranger installé en Macédoine. ${ }^{76}$ Le casque du type bonnet phrygien, avec l'inscription «basileōs Monouniou», trouve sur les bords du lac d'Ohrid et conservé au Musée de Berlin, est daté à l'époque hellénistique. ${ }^{77}$ Même si on considère ce nom comme illyrien, la valeur de ce témoignage quant à l'appartenance de Dassarétie à Illyrie est faible, étant donné que les objets voyagent plus que les noms. Les Macédoniens se disputaient avec des Illyriens sur ces régions frontalières, et il est normal qu'on trouve un objet d'équipement militaire. Selon premier éditeur (Th. Wiegand), vu le signe numérique «ny», le casque appartenait à un soldat. ${ }^{78}$

Tata et les nombreuses élargissement faites à l'aide de différents suffixes (Tataia, Tatia, Tatas, Tatais, Tatiane Tateis, Tates, etc) est un des noms les plus fréquents dans l'onomastique d'Asie Mineure et en conséquence considéré comme microasiatique par excellence. ${ }^{79}$ Pourtant, il n'est nullement attesté en Illyrie méridionale, hormis à Dyrrhachion. ${ }^{80}$ On a trouvé un exemple du nom féminin Tato dans chacune des deux colonies. ${ }^{81}$ Selon L. Robert, les noms Amia, Tata, Tatia etc. sont essentiellement «...de 'Lallnamen' d'origine indigène, mais que l'on peut dire d'apparence internationale, ayant leurs semblables ou leurs parallèles en toute langue..... ${ }^{82}$

La petite minorité des noms illyriens en Dasarétie méridionale (qui fait moins de $10 \%$ de tous les noms) est parlante. Il faut souligner que sur six noms prétendus illyriens, cinq ne sont pas du tout attestés dans des régions incontestablement illyriennes, hormis dans les deux colonies grecques Apollonie et Dyrrhachion. Or, leur population n'était pas majoritairement illyrienne, mais plutôt grecque et cosmopolite. Cela est démontrable surtout avec les listes des magistrats des colonies où la prépondérance des noms grecs est évidente. Sur 350, seulement 11 noms considérés illyriens sont portés par des monétaires. ${ }^{83}$ De ce fait la valeur de l'onomastique de ces villes portuaires pour déterminer comme illyriens les noms plus au moins obscurs et inhabituels, ne peut pas être concluante. Or, partout les noms indigènes dominent en dehors des grands centres urbains. ${ }^{84}$

${ }^{76}$ F. Papazoglou, Un témoignage inaperçu sur Monounios l'Illyrien, ŽAnt, XXI/1, 1971, p. 183, n. 12.

${ }^{77}$ F. Papazoglou, ŽAnt, XXI/1, p. 177-184, fig. 1 à la p. 180.

${ }^{78}$ cf. F. Papazoglou, op. cit., p. 182, n. 11.

${ }^{79}$ L. Zgusta, op. cit., nos 1517.

${ }^{80}$ CIGIME, nos 49, 391- 395.

${ }^{81}$ CIGIME, no 155 (Appolonie), 398 (Dyrrhachion).

${ }^{82}$ L. Robert, Noms indigènes, p. 348.

${ }^{83}$ M. Beauregard, op. cit., p. 103.

${ }^{84} \mathrm{Il}$ est intéressant de noter que même dans les centres secondaires de l'intérieur, dans les ateliers céramiques à Dimale, Klos, Byllis situés dans l'arrière pays d'Apollonia, l'onomastique est plus grecque qu'illyrienne cf. P. Cabanes, Les Illyriens des Bardylis à Genthios, Paris, 1988, p. 202-203. 
L'opinion que la partie nord de la Dassarétie ou la Penestianae terrae de l'antiquité, ${ }^{85}$ appartenait à la Mésie Supérieure, contrairement à la donnée de Ptolémée qui l'attribue à la Macédoine, ${ }^{86}$ a longtemps prévalue. A défaut de toute recherche archéologique et épigraphique, la seule inscription connue jusqu'à la II guerre mondiale étant en latin, certains savants ${ }^{87}$ avaient opté pour une correction des données de Ptolémée. Mais les inscriptions découvertes postérieurement ${ }^{88}$ confirment l'opinion que cette région faisaient partie de la Macédoine. ${ }^{89} \mathrm{~F}$. Papazoglou considérait cette région d'abord comme illyrienne, ${ }^{90}$ et plus tard comme faisant partie de la province macédonienne. ${ }^{91}$ Pour première opinion elle trouvait ses arguments dans les dires des auteurs anciens sur le désert illyrien créé par les Macédoniens pour prévenir les attaques des Dardaniens en Illyrie et en Macédoine, ${ }^{92}$ et le localisait sur le territoire des Pénestes, autour de la ville actuelle de Gostivar. ${ }^{93}$ Cependant, la description du trajet de la délégation envoyée par Persée chez Genthios, afin de conclure l'alliance contre les Romains, contredit une telle localisation. En décrivant le trajet Polybe écrit explicitement que les deux émissaires après avoir traversé Scardus mons voyageaient à travers de le désert illyrien eremos Illyris. ${ }^{94}$ De même Tite Live écrit que la délégation avait traversé la crête (transgresi iugum) de la Scardus mons (actuel Šar planina), et qu'après beaucoup de souffrances, elle est arrivée à Scodram ...tandem pervenerunt par le désert illyrien per Illyrici solitudines. ${ }^{95}$ De ces données s'ensuit que le désert illyrien ne se situait pas au sud de la Scardus mons mais à l'ouest, probablement dans la vallée de la rivière de Drim (Drilon). Cela est en

${ }^{85}$ Aujord'hui la plaine de Polog et le cours supérieur de la rivière Treska au nordouest de la R. de Macédoine. Sur le territoire des Penestes et localisation de la ville d’Uscana voir Н. Проева, За дасаретските племиња, ŽAnt, LXIV, 2014, pp. 165-180, resumé $180-181$, cartes $1-2$.

${ }^{86}$ Ptolemée, II, 16.

${ }^{87}$ N. Vulić, Severna granica antičke Makedonije, Strena Buliciana, Zagreb 1925, p. $242-243$.

${ }^{88}$ Н. Проева, Нови и ревидирани натписи од Полог и Кичевскиот крај, ŽAnt, XXXIX, 1989, p. 77-84 (résumé 83-84); I. Mikulčić., Teritorija Skupa, ŽAnt,XXI/2, 1971, p. 468-469.

89 Ћ. Трухелка, Археолошке белешке из јужне Србије, Гласник Скопског Научног Друштва, V, 1929, p. 72-73; N. Proeva, Onomastika i etnička struktura zapadnog dela SR Makedonije u antičko doba, GodCBI, XIX/27, 1991, p. 185-196, resumé 1961 98, Ead., ŽAnt, LXIV, 2014, pp. 166-167.

90 The Central Balkan Tribe in Pre-roman Time, Amsterdam, 1978, p. 191, carte no 2 et 3 à la page 188, 193 Ead., Sur la monnaie illyrienne au nom de $P H \Delta \Omega N$, ŽAnt, XXIV, 1974, p. 260.

${ }^{91}$ F. Papazoglu, The Central Balkan Tribes, p. 654, note 16; Ead., Les royaumes d'Illyrie et de Dardanie, M. Garašanin (dir.) Les Illyriens et les Albanais, Beograd, 1988, p. 193.

92 Polybios, XXVIII, 8, 1-5; T. Livius, XLIII, 20, 1.

${ }^{93}$ F. Papazoglu, Plemena, p. 130.

${ }^{94}$ Polybios XXVIII, 8.

${ }^{95}$ T. Livius, XLIII, 20. 
accord avec les données de Ptolémée qui écrivait que la frontière nordouest de la Macédoine commençait à Dyrrhachion et allait jusqu'au mont Scardus, ce qui veut dire qu'elle devrait longer la vallée de la Drim par laquelle les Dardaniens pouvaient facilement s'introduire en Illyrie. Strabon écrit que la frontière orientale des Illyriens était la rivière Drilon (Drim). ${ }^{96}$ En faveur de l'appartenance de la région des Pénestes à la Macédoine au moins à l'époque des Antigonides plaide le fait que dans la ville pénestienne d'Uscana était stationnée une garnison de soldats crétois. ${ }^{97}$ Notamment les cités crétoises étaient des alliées de la Macédoine. ${ }^{98}$ Les donnés sur cette région aux confins macédo-illyriens et dardaniens manquent pour les époques antérieures. L'intérêt sur cette région ne commence qu'avec l'arrivée des Romains. Les seules données sur les rapports des Pénestes avec les Illyriens sont celles de T. Live qui distingue les habitants d'Uscana et les Illyriens à deux reprises. La première à propos de reddition d'Uscana à Persée: Illyriorum cohors et Uscanenses se urbemque dedierunt; et la seconde à propos de la vente des prisonniers: Uscanensibus Illyrisque venditi. ${ }^{99}$ Malgré cela, l'opinion concernant l'appartenance illyriennes des Pénestes persiste toujours.

Les sources épigraphiques et surtout les données onomastiques oeuvrent en faveur de l'origine nord-macédonienne. Les noms attestés à présent sont: Alexandros, (H)Ameilos, Ammia, Anthimos, Apolodoros, Asandros, Getas, Genthiane, Eubia, Euridike, Eusebes, Kallityche, Lykos, Neikeratos, Nikanor, Nikephoros, Rhedon et Chares. ${ }^{100}$ La plupart de ces noms ne méritent pas de commentaire. Ce qui frappe à première vue, c'est la prépondérance de noms typiques de la Macédoine, et de la partie orientale de l'Empire romain et très rarement attestés dans la partie occidentale. ${ }^{101}$

Alexandros et Euridike sont des noms royaux fréquents chez les Argéades. L'anthroponyme Getas apparaît depuis le V s. av. n. e. comme le nom d'un basileus édonien, ${ }^{102}$ dont les légendes sur les monnaies

${ }^{96}$ Strabo, VII, 5 (316).

97 T. Livius, XCIII, 10, 1.

${ }^{98}$ Polybios, IV. 55. 1. Il nomme la lige comme l'alliance générale, cf. IV, 9, 4. Depuis Philippe V au moins les villes crétoises étaient devenues membres de la lige conclue par Antigone Doson en 224 av. n. ère.

${ }^{99}$ T. Livius, XCVIII, 18, 10 et XCIII, 19, 2.

${ }^{100}$ Н. Проева, ŽAnt, XXXIX, 1989, p. 77-84.

${ }^{101}$ Comme c'est cas surtout avec Anthimos, dont le pendant féminin est attesté en Cappadoce, Bull. ép., 1939, no 447.

${ }_{102}$ M. Price, Coins of the Macedonians, London, 1973, Pl. III/14. Il est presque inutile de dire que, quoique le nom n'est pas attesté en Thrace proprement dite, les chercheurs bulgares le considèrent comme thrace, ce qui est en accord avec leur théorie sur l'appartenance thrace des tribus édono-péoniennes. cf. D. Detschev, Die trakischen Sprachreste, Wien, 1957, p 103 sqq. J. Bousquet, Une épigramme funéraire grecque de Dardanie, ŽAnt, XXIV, 1974, p. 257 l'identifie avec l'ethnonyme. Les exemples réunis par D. Dana, Etudes sur les porteurs du nom ГETAC, Studii Clasice XXVIIXXXIX, 2001-2003, p. 93, qui attribue la région de Polog à la Mésie Supérieure. 
montrent les traits d'un vernaculaire i.e. génitif en $-a .{ }^{103}$ Le nom Getas n'est attesté que dans les colonies grecque de Dyrrachium et d'Apollonia $^{104}$ et pas du tout dans l'arrière pays, ce qui constitue un indice bien révélateur. Selon $\mathrm{C}$. de Simone ce nom ne peut être considéré comme sud-illyrien. ${ }^{105}$ Parmi ces noms des remarques plus détaillées appellent le noms: (H)Ameilos, Ammia, Genthiane et Rhedo, et sont susceptibles d'être illyriens.

Autant que j'ai pu le vérifier, (H)Ameilos n'est pas attesté en Macédoine. Le plus proche serait le nom féminin Ammeila ${ }^{106}$ et la forme latine Hamillus. ${ }^{107}$ Une Hamilla est lu sur une base de Thessalonique, aujourd'hui perdue. ${ }^{108}$ A propos de cette inscription J. H. Mordtman a écrit qu'Ammeila est formée de la racine macédonienne avec le suffixe -eil(l)a. ${ }^{109}$ Sur l'inscription de manumission de Leukopetra, les éditeurs corrigent Amilla en Ammila, ce qui est lecture arbitraire, et le considèrent comme diminutif d'Ammia. ${ }^{110}$ Selon $\mathrm{O}$. Masson le nom Ammilla attesté une fois en Apollonie (de meme qu'Annaia), est difficile à classer et à caractériser l'origine, il les considère plutôt comme «Lallnamen». ${ }^{111}$ A. Tataki range les noms Amila ou Ammila dans le même groupe que Ammia, Amian, Amianos. ${ }^{112}$

Ammia est un nom qui était considéré comme micro-asiatique. ${ }^{113}$ Mais, à part de l'Asie Mineure, il est largement répandu dans toutes les régions de Macédoine, ainsi que dans la zone culturelle grecque, ${ }^{114}$ mais pas dans la partie sud-illyrienne, donc il ne peut être considéré comme illyrien, non plus. Ce nom figure sur les gradins du théâtre de Lychnidos. ${ }^{115}$ La parenté onomastique entre la Macédoine et l'Asie

${ }^{103}$ P. Perdrizet., Contribution à l'étude du macédonien, $B C H 35,1911,120-131$.

${ }^{104}$ CIGIME, I, 1 nos 155, 306, 424 avec des patronymes grecs. CIGIME, I, 2 no 65 , le patronyme Plator (voir plus haut) montre que Getas appartient aux vielles couches balkanique le substrat pré-illyrien et pré-thrace. F. Papazoglu, Sur quelques noms «thraces» en Illyrie, GodCBI, 12, 1974, p. 67. Sur le sens flottant du terme Thrace chez les auteurs anciens et l'usage sans précaution par les modernes voir N. Proeva, On the Names of Thracia and Eastern Macedonia, in Kratistos Volume in honour of Professor Peter Delev, Sofia, 2017, p. 75-82.

${ }^{105}$ C. De Simone, Grecs et Illyriens, App. C. VI, p. 71/2.

${ }^{106}$ L. Zgusta, op. cit., no 57-26.

${ }^{107}$ H. Solin, Beiträgre zur Kenntnis der griechischen Personennamen in Rom, Commentationes humanorum literarum,, t. CXVIII, 1971, p. 80.

${ }^{108} I G$ X, II, 2, no 462.

109 J. H. Mordtman, Zur Epigraphik von Kyzios II, Athenische Mitteilungen, 7, 1882, p. 257.

${ }^{110} \mathrm{Ph}$. Petsas-M. Hatzopoulos-L. Gounaropoulos-P. Paschidis., Inscriptions de sanctuaire de la Mère de dieux autochtones de Leukopetra (Macédoine), Meletemata no. 28 , Athenes 2000 , no 15 .

111 O. Masson, Encore les noms grecs et les noms illyriens à Apollonia et Dyrrhachion, in Grecs et Illyriens, p. 77.

${ }^{112}$ Ancient Beroia, prosopography and society, Meletemata 8, Athenes 1988, p. 400.

${ }^{113}$ L. Zgusta., op. cit., no 57.

${ }^{114} \mathrm{Il}$ suffit de feuilleter les index des corpus épigraphiques publiés à nos jours.

${ }^{115}$ IG X, II, 2, no 401. 
Mineure, notamment Phrygie et Bithynie est bien connu, ${ }^{116}$ et il est évident que ce nom est d'origine brygienne.

Concernant le nom Genthiane il faut tout de suite souligner qu'il n'est pas attesté en Illyrie du sud, ${ }^{117}$ et que son pendant masculin l'est en Dassarétie (près de Podgradec). ${ }^{118}$ En tant que cognomen Genthianos apparaît une fois à Apollonia. ${ }^{119}$ Les noms au radical -genth (Gentheis, Genthis, Genthena) étaient considérés aussi comme illyriens. Il en était de même pour les noms avec la graphie latine -gent (Gentione, Gentilla). Ces noms ne sont pas attestés en Illyrie méridionale et rarement en Illyrie du nord. Dans la péninsule Apennine les formes Gentis et Gentia apparaissent toujours en tant que gentilice. ${ }^{120}$ De ce fait ces noms sont considérés par les linguistes comme romains. Du radical -genth et avec différents suffixes tels que -(e)as, -is, -eis, anos, -ne, -ena, -illa étaient construites différentes formes (masculines et féminines), telles Gentheas, Genthis, Gentheis, Genthianos, Genthiane, Genthena, Gentilla, qui sont d'apparence plutôt grecque qu'illyrienne. En faveur de cela, il se trouve que ces noms ne sont attestés que dans les colonies grecques (comme cognomen, Genthis est attesté à Apollonia ${ }^{121}$ ) et non pas dans l'arrière pays chez les gens simples.

Le nom Rhedon est très rare et longtemps il n'était connu que par les monnaies de Lissos, ${ }^{122}$ et longtemps après sur une inscription trouvée au village de Lešok, près de Tetovo, ${ }^{123}$ sur le territoire des Pénestes. Plusieurs opinions sur son identité ont été exprimées: divinité ${ }^{124}$ (Hermes), divinité ou héros illyrien, ${ }^{125}$ dynaste (Genthios), ${ }^{126}$ magistrat

${ }^{116}$ F. Papazoglou., Structure ethnique et sociales dans les Balkans, Actes du VII congrès intern. d'épigraphie grecque et latine, 1979, p. 151-152.

${ }^{117}$ Une Genthena est attestée en Dardanie, mais l'onomastique dardanienne n'est pas illyrienne dans le sens stricto sensu.

${ }^{118}$ V. Toçi, Studia Albanica, 9/1, 1972, p.84 ; C. De Simone, Grecs et Illyriens, p. 55.

${ }^{119}$ CIGIME, I, 2 Apollonia, no 189.

${ }^{120}$ Les exemples rassemblés par R. Katičić, ŽAnt, XII, 1962, p. 105.

${ }^{121}$ CIGIME, no 189.

$122 \mathrm{~J}$. Brunschmid, Die inschriften und munzen der griechischen städte dalmatiens, Wien, 1898, p. 74, no 3, T. VI, 93.

${ }^{123}$ J. Bousquet, ŽAnt, XXIV, 1974, p. 255-257.

${ }^{124} \mathrm{~J}$. Brunschmid, loc. cit.

${ }^{125}$ H. Ceka, Question de numismatique illyrienne, Tirana, 1972, p. 162-169; Id. Le buste du roi Genthios ou une figure de mythologie illyrienne? Iliria, VI, 1976, p. $145 / 6$.

126 S. Islami, Monnayage de Skodra, Lissos et Genthios (essai d'une révision du problème), Studia Albanica I, 1966, p. 236, Pl. X, 3-4 ; D. Rendić-Miočević, Ilirski vladarski novci u Arheološkom muzeju u Zagrebu, VAM, VI/VII, Zagreb, 1972/3, p. 258-260) quoiqu'il hésite entre roi Genthios ou Hermes opte plutôt pour le roi, mais n'exclut pas qu'une divinité locale est représentée (p. 266); dans un travail postérieur, l'auteur considère que le nom désigne le magistrat monétaire mais avance l'idée que l'effigie représente une divinité locale ou héros, Id. Novi prilozi pitanju Rhedon-emisija Lješke kovnice, VAM 3. s., XVIII, Zagreb 1985, pp. 45-56. 
monétaire ${ }^{127}$ ou gouverneur de la première région illyrienne, ${ }^{128}$ (définie par les Romains après 167, av. n. ère). On a pu trancher la question avec certitude grâce à l'inscription - graffite en latin inscrite sur le rocher de la grotte Porcinara près de la ville Leuca en Calabre, qui porte la dédicace des équipages de deux navires nommés Medaurus et Rhédon. ${ }^{129}$ Or, depuis longtemps on sait que Médaure était vénéré en tant que héros /ancêtre de la ville Risinium, Rhizon (actuelle ville Risan en Montenegro) sous forme d'un cavalier. Deux inscriptions votives trouvées à Lambèse en Afrique, lui étaient consacrées. ${ }^{30}$ Cela montre que Rhedon doit être soit le nom d'un héros, soit celui d'une divinité. A l'encontre de la thèse selon laquelle il s'agirait d'une effigie divine est le fait que la même image: la tête coiffée avec petasos, kausia ou coiffure, mais sans nom, se trouve sur les monnaies des tribus de Daorses, ${ }^{131}$ Labeates, ${ }^{132}$ et de la ville Skodra. ${ }^{133}$ Comme il est clair qu'il s'agit de divinité ou d'un héros, cela signifie que le nom Rhedon qui figure sur l'inscription de Lešok est un nom divin - nomen sacrum.

Quant à son origine, certains le considèrent comme grec, ${ }^{134} \mathrm{~d}$ 'autres comme illyrien, ${ }^{135}$ ou encore celte (à part de l'ethnique Redones, ${ }^{136}$ le Redonum civitates est attesté épigraphiquement). ${ }^{137}$ En faveur de l'origine celte on peut évoquer les gentilices Raedonius, Raedonia, attestés en contexte de noms indigènes en Italie septentrionale. ${ }^{138}$ En proposant une étymologie du nom, M. Petruševski penche plutôt pour une attribution illyrienne que celte. ${ }^{139}$ Sachant que les noms à la racine * red-ne sont pas attestés chez les Illyriens et que hormis sur les monnaies de Lissos, on ne trouve point le nom Rhedon en Illyrie méridionale, on ne peut pas considérer ce nom comme illyrien. Il est plus prudent,

${ }^{127}$ F. Papazoglou, Sur la monnaie illyrienne au nom de $\mathrm{PH} \Delta \Omega \mathrm{N}, \check{Z} A n t$, XXIV, 1974, p. 259; O. Masson, Variétés thessaliennes, RPh, LIV/2, 1980, p. 230.

${ }_{128}$ B. Jubani, Monnaies illyriens à l'éthnikon de $\Lambda$ ABIATAN découvertes à $\mathrm{Ku}$ kës, Studia Albanica IX, 1972, p. 72.

${ }^{129} A E$, 1979, no 199: I(ovi) O(ptimo) M(aximo)/C(aius). Cordius Aqui/linus vot(um) sol(vit)/ cum pleroma(te) Rhedo[n]is et Me[d]aur(i).

${ }^{130}$ CIL VIII, 2581 = ILS 4881.

${ }^{131}$ Dj. Basler, Novćić plemena Daorsa, Glasnik Zemaljskog Muzeja XXVI, 1971, 333.

132 B. Jubani, op. cit. p. 75, no 1-4, Pl. II-IV.

${ }^{133} \mathrm{~S}$. Islami, op. cit. $\mathrm{Pl}$. I/1-2.

${ }^{134}$ W. Pape, Wörterbuch der griechischen Eigennamen, Braunschweig, 1991, s.v. Une Rhedeta Dioskouridou est connue en Thessalie par l'affranchissement de son esclave, cf. B. Helly, Acte d'affranchissement thessalien, $B C H$, 99/1, 1975, p. 127-129, no 2.

135 O. Masson, Variétés thessaliennes, $R P h$, LIV/2, 1980, p. 230 ; Id. Quelques noms illyriens, in L'Illyrie et l'Epire dans l'Antiquité, I, p. 117, l'auteur considère la région comme appartenant a Dardanie.

136 Ptolemée, II, 8, 12.

${ }^{137}$ Cf. $A E 1982$, no 55-57.

${ }^{138} A E, 1946$, no 220 (la région Anaunia).

139 M. Petruševski, ILLYR. PH $\Delta \Omega \mathrm{N}=*$ REG-ON-(CF. SSCR RAJAN)?, ŽAnt, XXIV, 1974, p. 260. 
pour le moment, de le classer parmi les noms paléobalkaniques, de même que le nom Baton. ${ }^{140}$ Cela d'autant plus que l'usage de donner des noms divins - nomina sacra, aux mortels n'étaient pas connu chez les Illyriens et les Thraces, ${ }^{141}$ parmi lesquels les noms théophores étaient en usage. ${ }^{142}$ Nomina sacra étant répandues en Asie Mineure et en Macédoine, ${ }^{143}$ il s'agit d'une coutume ancienne et autochtone, qui n'a pas été supprimée ni par l'hellénisation ni par la romanisation.

Pour conclure, les noms prétendument illyriens en Dassarétie sont attestés dans un contexte onomastique qui n'est point illyrien, et apparemment n'appartiennent pas à la lexique illyrienne et au fonds onomastique illyrien. Cela nous invite à la nécessité de restreindre l'appellation «illyrienne». Certains noms qui sont communs en Macédoine, en Illyrie méridionale et partiellement en Dardanie, appartiennent manifestement à des couches les plus anciennes. La ressemblance onomastique entre l'Illyrie méridionale et la Dassarétie est due à l'ancienne couche brygienne. Il suffit de mentionner un autre nom édonien, Dryas, le nom du père du roi édonien Lykurgos (Iliad, Z, 130), attesté deux fois à Ohrid, ${ }^{144}$ ce qui est révélateur de l'origine édono-brigyenne, et non pas illyrienne de ces noms et en conséquence des ces tribus. ${ }^{145}$ Les Brygoi sont mentionnées par les sources anciennes en toute la Macédoine (du Strymon et le golfe Thermaique à l'est jusqu'à l'Erigon à l'ouest), ainsi que dans l'arrière pays de Dyrrhachion. ${ }^{146}$ Etienne de Byzance avait défini les Brygoi comme tribu macédonienne. ${ }^{147} \mathrm{Ces}$ noms, ainsi que les monuments funéraires découverts dans la partie occidentale de la République de Macédoine, par leur caractéristique typologiques ${ }^{148}$ et onomastiques donnent raison à Ptolémée dans le sens que la région de la Dassarétie faisait partie de la Macédoine et non pas de la Mésie Supérieure et que les tribus en question n'étaient pas d'origine illyrienne. ${ }^{149}$ Les éléments communs à l'onomastique illyrienne

${ }^{140}$ Le nom a été étudie par Katičić R., Drei altbalkanische Nomina sacra, GodCBI, IX/7, Sarajevo, 1972, p. 105-120.

${ }^{141}$ F. Papazoglou, Deorum nomina hominibus imposita, Zbornik Filozofskog Fakulteta XIV/1, Beograd, 1979, p. 13.

${ }^{142}$ D. Rendić-Miočević, VAM 3. s., XVIII, 1985, pp. 55/56 qualifie nom Rhedon comme un anthroponyme théoforme.

${ }^{143}$ F. Papazoglou, op, cit., p. 16.

${ }^{144} I G$ X, II, 2 nos 369, 376.

${ }^{145}$ Nombreux exemples de Dryas en Asie Mineure cf. L. Zgusta, op. cit. 315-3.

${ }^{146}$ Appianus, Bel. Civ. II, 39; Strabo, VII, 5, 8, 9 (c. 321) et VII, fr. 25. = VII, 326. Le nom Brygos une fois est attesté à Dyrrhachion, CIGIME, I, 1, no 144.

${ }^{147}$ Steph. Byz. s.v. Bryx, et la ville Brygias mentionnée par Hésichios est notée dans l'Itinéraire Hierosolumitanum, 607, 2. La migration des Brygoi de Macédoine en Asie Mineure est confirmée par les analogies entre l'onomastique de ces deux régions et par la quantité des noms brygiens qui ont survécu en Macédoine jusqu'à la fin de l'antiquité.

${ }^{148}$ I. Mikulčić, ŽAnt XXI/2, 1971, p. 468-69, fig. 1, 4, 5.

${ }^{149}$ La présence de noms d'origine non illyrienne, mais adoptés par les Illyriens et par la suite répandus chez eux, tels que Ammia, Anna, Dazos, Dazaios, Genthios, Genthena etc. dans d'autres régions, ne prouve pas qu'elles soient illyriennes. Comme on 
et macédonienne sont dus non seulement à la couche brygienne, mais aussi aux relations tant politiques ${ }^{150}$ que culturelles entre la partie méridionale de l'Illyrie et la partie occidentale de la Macédoine, et révèle les contacts entre zones illyriennes et macédoniennes, qui depuis toujours, ont été un lieu de rencontre des civilisations.

Cette analyse a montré que les noms prétendument illyriens que l'on trouve dans les colonies grecques, ne peuvent être considérés comme tels, d'autant plus qu'ils ne sont pas si courants en pays incontestablement illyrien. La non prise en compte de ce fait fausse les résultats des recherches menées.

\section{ABREVIATIONS}

$\mathrm{AE}=$ Année Epigraphique

Bull. Ep. = Bulletin Epigraphique, Revue des études grecques, Paris

CIGIME $=$ CABANES P. (dir.), Corpus des Inscriptions grecques d'Illyrie méridionale et d'Epire, vol. I, 1 : Inscriptions d'Epidamne-Dyrrhachion, Paris, 1995; vol. I, 2 : Inscriptions d'Appolonia d'Illyrie, Paris, 1997; vol. 2,2 Inscriptions de Boutrothos, Paris, 2007

EAM = Ridzakis, Th. i Touratsoglou, I. Epigraphes ano Makedonias, Athens, 1985

$\mathrm{EKM}=$ Gounaropoulou, L.- Hatzopoulou, M. B., Epigraphes kato Makedonias, vol I, Epigraphes Beroias, Athens 1998

GodCBI $=$ Godišnjak Centra za balkanološka ispitivanja, Sarajevo

IG X, II, 2 = PAPAZOGLOU F. (ed), IG X, pars II Inscriptiones Macedoniae, fasciculus II Inscriptiones Macedoniae Septentrionalis : Inscriptiones Lyncestis, Heracleae, Pelagoniae, Derriopi, Lychnidi, Berolini, 1999

$\mathrm{RPh}=$ Revue de philologie, de littérature et d'histoire anciennes, Paris

$\mathrm{RE}=$ Pauly-Wissowa-Kroll, Realencyklopädie der classischen Altertumswissenchaft

VAM = Vjesnik Arheološkog Muzeja, Zagreb

ŽAnt $=$ Živa Antika Skopje

l'a vu, certains noms sont d'origine messapienne, d'autre d'origine brygienne. Un Ammia est appelée Illyria sur une inscription de Delphes, cf. H. Collitz-F. Bechtel, op. cit.

${ }^{150}$ Il ne faut pas oublier le donné de T. Live, XLV, 30. 5, sur les collons illyriens en III ème région de Macédoine à l'époque des Antigonides. A Edessa on a trouvé une épitaphe pour Gen[th]ios érigée par sa femme Apollonia (Bull.ép. 1974, 330), à Pella une Genthena fille d'un Magel[i]tou (Bull.ép. 1990, no 464), et certain Dazaios (Bull. ép. 196, 247) sont attestés. 